\title{
El karst de la Sierra de Segura: Propuesta de un itinerario didáctico
}

\author{
Begoña LoPez LIMIA
}

\section{INTRODUCCIÓN}

En este trabajo se establece un recorrido de campo, basado en el análisis de casos concretos, como ejemplos didácticos para el estudio del paisaje kárstico, resaltando así la importancia de los trabajos de campo como apoyo al estudio teórico de los fenómenos geográficos.

El presente itinerario propone ocho paradas idóneas para el conocimiento de este paisaje y la aplicación de distintas técnicas de análisis geográfico. Los ejemplos están desarrollados esquemáticamente, incluyendo una descripción de las formas observadas y una actividad complementaria a realizar en cada parada, aunque éstas son indicativas, pudiendo modificarse de acuerdo a las características de cada grupo de alumnos.

Para su ejecución se ha elegido un sector de la Sierra de Segura. El gran desarrollo alcanzado por los fenómenos kársticos en ella, hacen de esta sierra un lugar idóneo para el establecimiento de recorridos didácticos en el sureste de España.

El itinerario está dirigido, tanto por el fenómeno geográfico analizado, como por las actividades propuestas, a alumnos de niveles universitarios. 


\section{SITUACIÓN GEOGRÁFICA Y GEOLÓGICA}

Localizada en parte de las provincias de Granada, Jaen y Albacete, la Sierra de Segura se extiende en dirección SW-NE, quedando limitada al $\mathrm{N}$ por la sierra del Calar del Mundo, donde se desarrolla uno de los aparatos kársticos más complejos de esta sierra. El límite meridional se establece en el contacto con la cuenca del río Castril. Hacia el W. una alineación montañosa de dirección SW-NE, cuyo punto culminante se encuentra a $1.993 \mathrm{~m}$ (Banderillas), constituye la divisoria de aguas entre los ríos Segura y Guadalquivir.

Es en la cuenca alta del río Segura, a una altitud media elevada $(1.450 \mathrm{~m})$, donde se desarrolla el itinerario, comprendiendo parte de las hojas 22-36 (Santiago de la Espada), 22-35 (Orcera) y 23-35 (Yetas de Abajo) del Mapa Topográfico Nacional, escala 1/50.000 del IGE.

El área se caracteriza por una gran evolución de las formas kársticas, representadas por lo que localmente se conoce como "calares". Geomorfológicamente son zonas de absorción muy desarrolladas. En esta parte de la cuenca del Segura se ubican los calares del Cobo (cuenca del arroyo de las Gorgollitas), las Palomas y Pinar Negro (cuenca de absorción del nacimiento del río Segura).

Geológicamente el sector se inserta en el dominio Prebético Interno. Los términos representados, correspondientes al cretácico y terciario, son fundamentalmente carbonatados, aunque existen algunos episodios detríticos durante el Albense (arenas y margas de facies Utrillas), el Paleógeno y el Neógeno (arenas y limos) (García Hernández et al., 1979).

Tectónicamente el área está afectada por una estructura de plegamiento relativamente suave en general y tres grandes conjuntos de fallas: fallas inversas de dirección $\mathrm{N} 35-40^{\circ} \mathrm{E}$; fallas de desgarre $\left(\mathrm{N} 120^{\circ} \mathrm{E}\right)$ que interrumpen y desplazan a las fracturas anteriores y fallas normales, que se distribuyen en dos grupos, de los cuales el más importante es aquel en que las unidades se disponen paralelamente a la dirección de los pliegues.

\section{ITINERARIO}

El recorrido se inicia en Yeste, penetrando en el sector por el NE (Figura 1). 
El karst de la Sierra de Segura: Propuesta de un itinerario didáctico

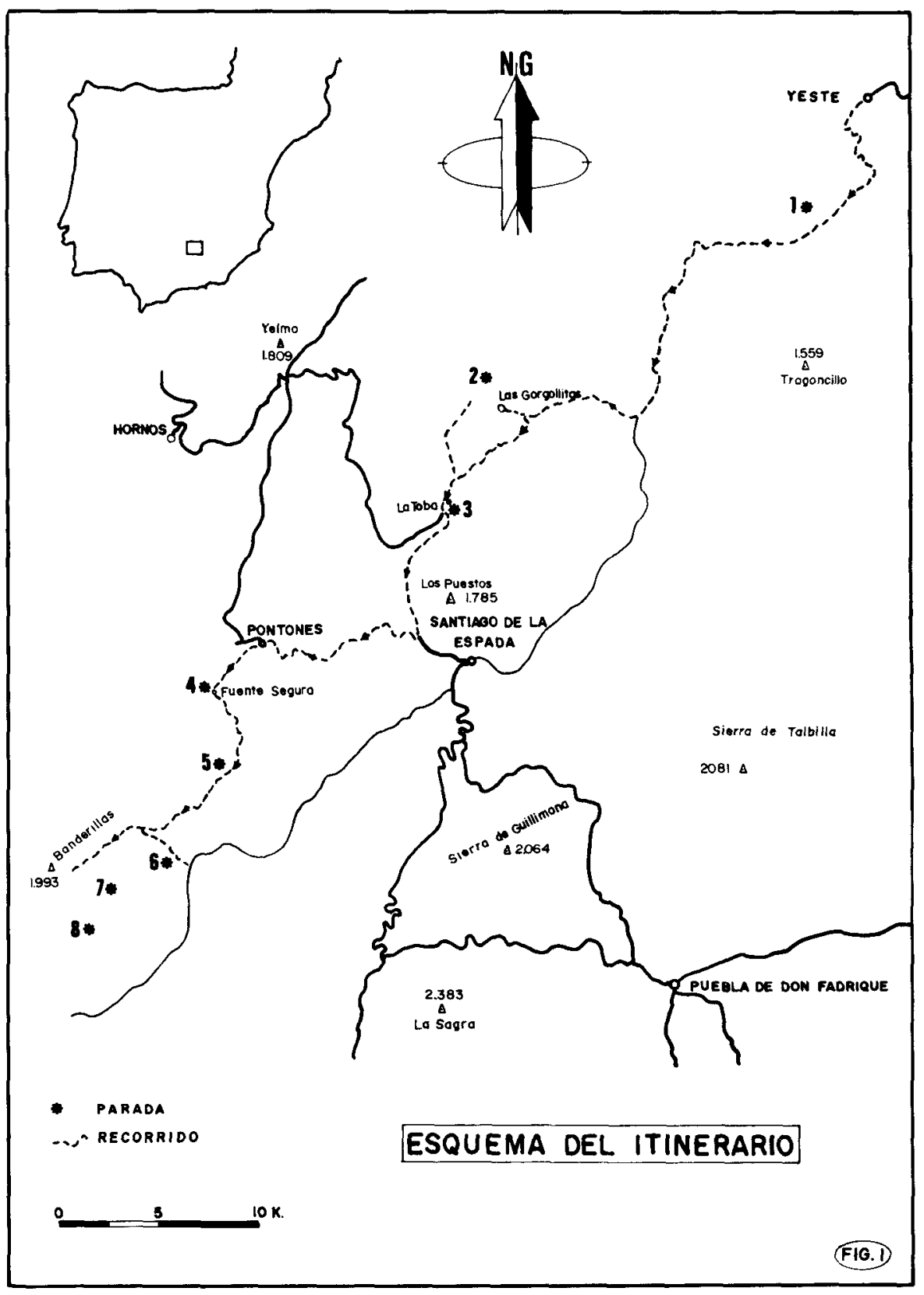




\section{Parada n. ${ }^{\circ} 1$}

Desde Yeste se toma la carretera local que conduce hacia la aldea de la Graya. A la altura de esta población, en la margen derecha de la carretera se efectúa la primera parada. Su objetivo es mostrar un ejemplo de surgencia fosil, en el que se pueden establecer relaciones entre la evolución de la red fluvial general y la evolución de la cavidad.

Se sitúa a $150 \mathrm{~m}$ por encima del nivel actual del río Segura con el que se halla conectada por medio de un pequeño arroyo. En la génesis y evolución de la cavidad se reconoce la acción de dos procesos distintos. En un primer estadio la cueva actuó como surgencia de las aguas recogidas en la cuenca superior. Posteriormente, la profundización general de la red hidrográfica, supone el abandono de esta surgencia y en su evolución van a predominar los procesos clásticos, favorecidos por la intensa fracturación a que está sometida la zona y por el buzamiento de los estratos.

Objetivos:

- Concepto de surgencia activa y fósil.

Actividades:

- Medir el buzamiento y dirección de los estratos.

- Realizar un croquis reflejando la posición de la surgencia y del río.

\section{Parada n. ${ }^{\circ} 2$}

Continuando por la misma carretera en dirección al embalse de Anchuricas, a $30 \mathrm{~km}$, se toma un camino forestal a la izquierda de la carretera que conduce a las Gorgollitas, donde tiene lugar el nacimiento del arroyo de este mismo nombre.

El conjunto (la surgencia junto a su cuenca de alimentación constituida por el Calar del Cobo) es un interesante ejemplo de aparato kárstico en el que son reconocibles la mayoria de sus partes y que permite observar las relaciones entre las tres zonas que componen un karst: absorción, conducción y surgencia.

La Loma del Calar del Cobo es un sinclinal colgado a una altitud media de $1.600 \mathrm{~m}$, donde se ubica una sucesión de tres poljes (entre los que destaca el más septentrional), establecidos en las calizas bioclásticas 
del mioceno medio. Morfológicamente la depresión más septentrional es un polje kárstico cerrado: llanura formada por un depósito horizontal de aluviones fluviales situado en una depresión kárstica cerrada, con drenaje subterráneo (Hernánz, 1968). El drenaje se efectúa a partir del ponor del polje, que es penetrable hasta una profundidad de $75 \mathrm{~m}$. La proximidad de la surgencia de las Gorgollitas $(400 \mathrm{~m})$ y la dirección de la sima hace pensar en una conexión directa entre el polje y la surgencia.

Objetivos:

- Concepto de polje.

- Concepto de sistema kárstico.

Actividades:

- Realizar un corte estratigráfico en el mapa geológico, observando la naturaleza de los materiales en los que se desarrollan las distintas partes del aparato kárstico.

- Dibujar en un bloque diagrama las zonas de un sistema kárstico.

\section{Parada $\mathbf{n}^{\circ} 3$}

De vuelta a la carretera se continúa hacia el Sur hasta llegar a la aldea de La Toba, atravesando el embalse de Anchuricas. El motivo de esta parada es observar la relación entre una surgencia kárstica y un depósito de travertinos.

La surgencia de La Toba se establece en el contacto entre los materiales carbonatados del cretácico superior y los materiales impermeables del Albense (Figura 2). El cambio en las condiciones ambientales (presión y temperatura) del caudal de agua circulante, provoca en las proximidades de la surgencia la formación de travertinos: calizas originadas en las aguas continentales por depósito del carbonato cálcico al desprenderse el anhídrido carbónico del agua.

Objetivos:

- Comprender el proceso que origina los travertinos.

- Establecer las distintas clasificaciones de rocas carbonatadas.

Actividades:

- Levantamiento topográfico de la cavidad (planta y alzado de la parte accesible). 




Parada n. ${ }^{\circ} 4$

Desde La Toba un camino de reciente construcción desemboca a 5 $\mathrm{km}$ de Santiago de la Espada. A partir de este pueblo se toma una carretera local hasta el desvío de Fuente de Segura, cercano a Pontones.

Las surgencias más frecuentes de la zona están asociadas a la existencia de acuíferos colgados, desarrollados en el contacto entre materiales permeables e impermeables. Sin embargo, el caso del río Segura es distinto, y su nacimiento está ligado a la presencia de una falla inversa que superpone parte del complejo dolomítico del cretácico superior a los materiales margosos del helveciense (Dabrio González, 1973).

Las escorrentías recogidas en la cuenca de alimentación desaguan a través de un sifón que ha sido explorado en su tramo inicial y que se dirige hacia el SE, conectando con el polje de Cañada Cruz, situado en esta misma dirección $\mathbf{3 0 0} \mathrm{m}$ por encima del nacimiento del Segura.

Objetivos:

- Distinguir entre circulación libre y a presión.

- Conocer los distintos tipos de conductos kársticos. 
Actividades:

- Tomar muestras de agua para medir su Ph y temperatura.

\section{Parada n..$^{\circ} 5$}

Desde la parada anterior un camino forestal asciende durante $2,5 \mathrm{~km}$ hasta el polje de Cañada Cruz, desarrollado en los materiales carbonatados de edad senonense medio-superior. Ha aprovechado para su expansión la presencia de una gran falla normal.

El fondo de la depresión presenta distintos niveles ocasionados por la presencia de un sistema de conos que tapizan el polje en ambas márgenes (Figura 3).

Hidrológicamente puede definirse como un polje intermitente, ya que durante los periodos de deshielo parte de él se inunda. Generalmente

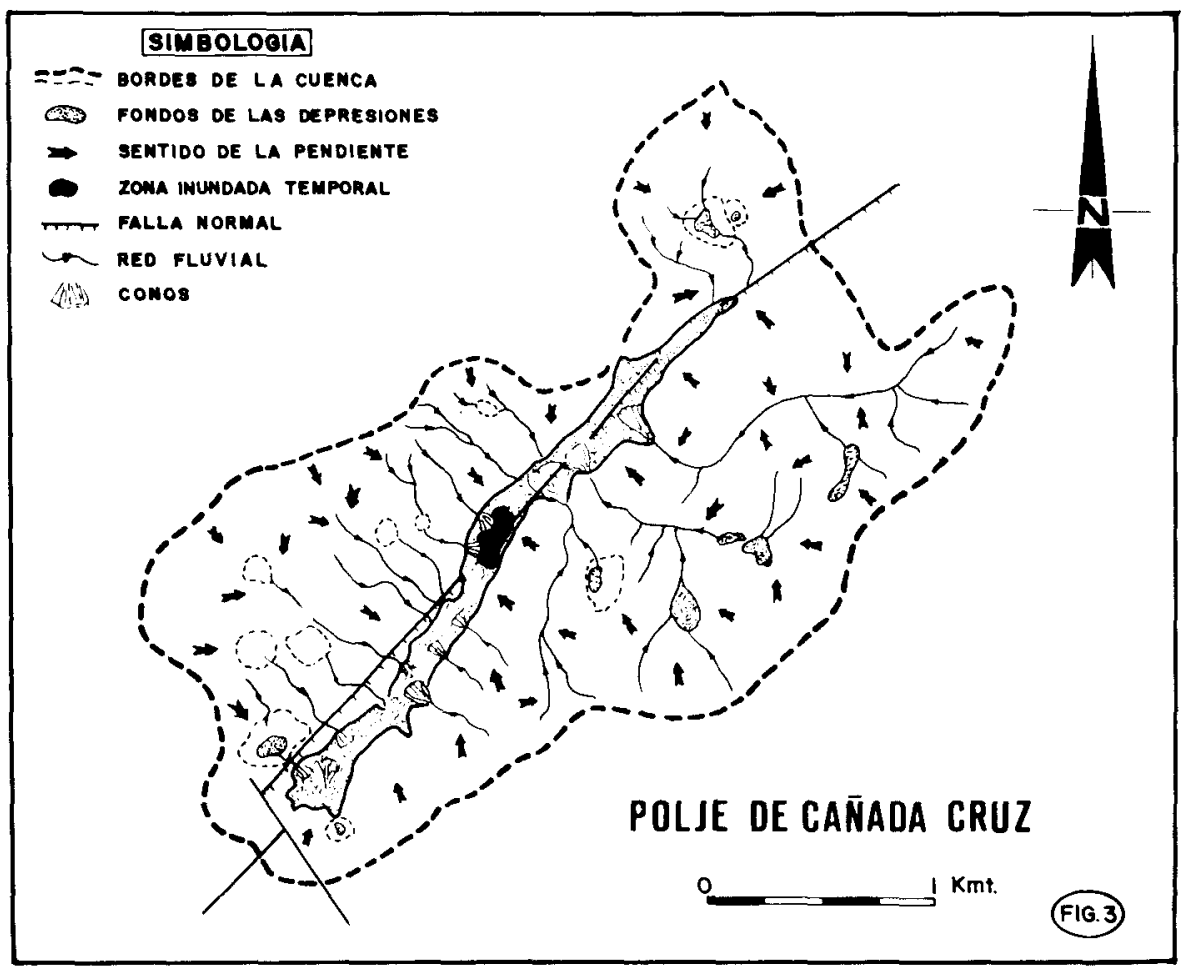


esta inundación se corresponde con un mayor caudal del río Segura, to que corrobora la conexión entre la surgencia y el polje.

Cabe aprovechar esta parada para observar algunos ejemplos de capturas de dolinas en la vertiente $W$ de la depresión.

Objetivos:

- Establecer las relaciones entre nivel freático-fondo de los poljes.

- Conocer los distintos tipos de poljes y sus características.

Actividades:

- Delimitar en la fotografía aérea la cuenca de alimentación del polje.

- Diferenciar entre los sedimentos propios del polje y los aportes alóctonos.

\section{Parada.$^{\circ} 6$}

La sima de hundimiento objeto de esta parada se localiza en el extremo sur de una gran uvala, a la que se accede por el camino que, desde Cañada Cruz, conduce a los campos de Hernán Pelea.

La sima, de $16 \mathrm{~m}$ de profundidad, consta de un primer tramo vertical y dos galerias descendentes paralelas entre sí (Figura 4). Genéticamente es una sima de hundimiento, en la cual el agua ha ido excavando los vanos inferiores hasta producir un vacio que provoca el hundimiento de los tramos superiores (Geze, 1974). La disolución ha sido favorecida por la presencia de fracturas de dirección $\mathrm{N} 70^{\circ} \mathrm{E}$, condicionando la orientación de la cavidad. La boca de la sima constituye el sumidero de la gran uvala.

Objetivos:

- Concepto de uvala.

- Distinguir entre proceso de disolución directa e inversa en la génesis de las simas.

Actividades:

- Reconstrucción del proceso kárstico que origina el hundimiento. 


\section{S.2 SIMA DE hUNDIMIENTO}

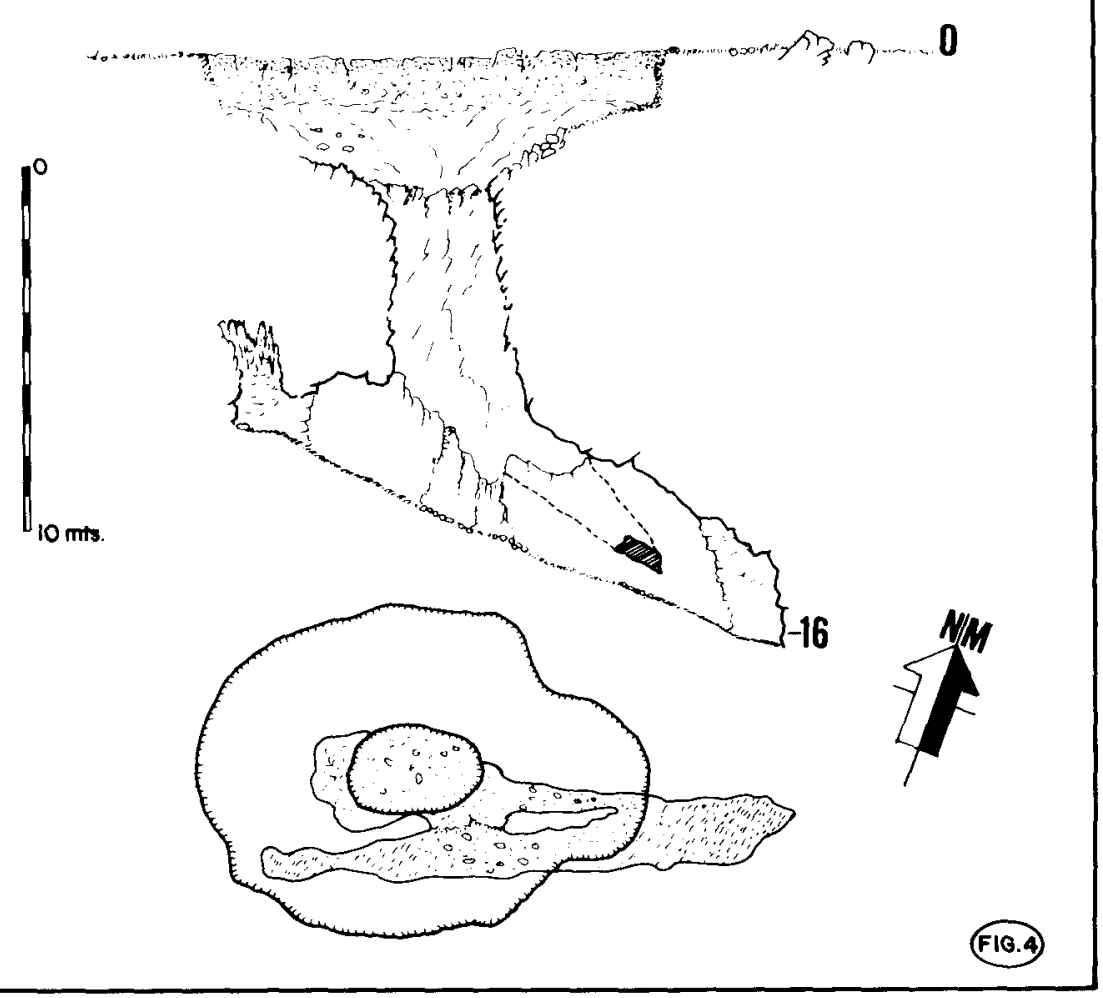

\section{Parada . $^{\circ} 7$}

Se desciende hacia el $W$ en dirección al cortijo de Pinar Negro situado al pie del Banderillas. Ascendiendo desde la casa unos $200 \mathrm{~m}$ por la cuerda del Banderillas, se obtiene una buena panorámica del campo de dolinas.

El proceso de dolinización es el más característico de Pinar Negro, contabilizándose un total de 1880 formas simples, con una densidad de 80 dolinas por $\mathrm{km}^{2}$. El campo de dolinas, establecido en los materiales carbonatados del cenomanense se estructura según los principales acci-

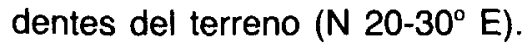


Genéticamente se reconocen en el área tres tipos de depresiones simples (López Limia, 1985): dolinas de solución, que son las más numerosas, dolinas de subsidencia y dolinas de hundimiento (Figura 5). Su disposición recuerda la estructura de los cockpit tropicales.

Objetivos:

- Concepto de karst tropical - karst templado.

- El proceso de dolinización en la evolución de un karst.

Actividades:

- En un bloque diagrama reflejar el origen de los tres tipos de dolinas.

- A partir de la fotografía aérea medir las orientaciones de los ejes mayores de las dolinas y de las fracturas, comparando los resultados.

\section{Parada $\mathbf{n}^{\circ} 8$}

Desde el cortijo de Pinar Negro hacia el sur se llega a la Casa de la Sima. A pocos metros de ésta se localiza el último punto del itinerario.

Las rocas carbonatadas están dispuestas en estratos subhorizontales, altamente fracturados. Estas condiciones han favorecido la formación de un lapiaz de diaclasas o kluftkarren: hendiduras agrandadas por la corrosión de las diaclasas (Sweeting, 1973). Formando parte de este lapiaz se obsenvan algunos ejemplos de corredores o bogaces, con una profundidad de hasta $5 \mathrm{~m}$ y un metro de anchura.

Objetivos:

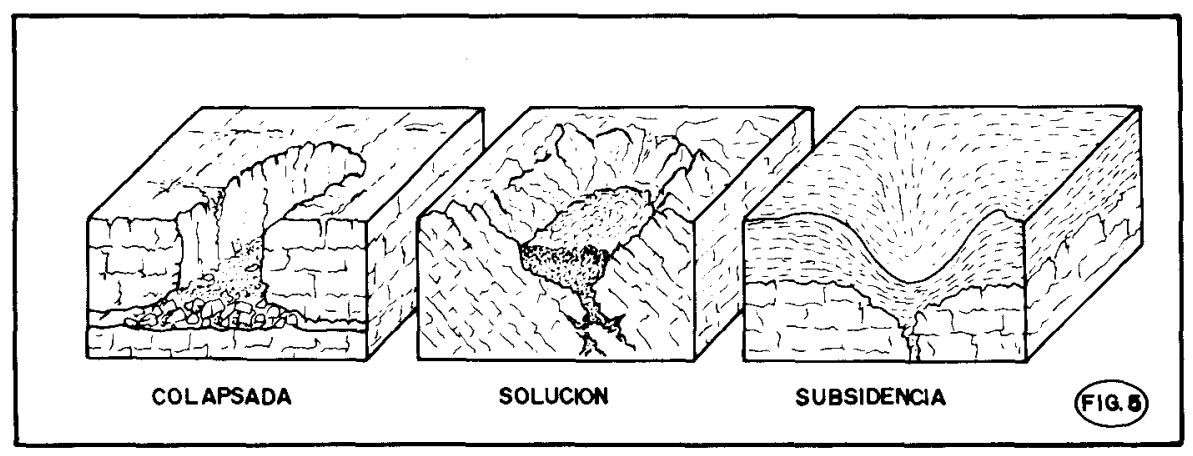


- Concepto de lapiaz y su importancia como forma de absorción.

- Observar las relaciones entre lapiaz-litología-fases de formación. Actividades:

- Elaborar una ficha tipo para su análisis morfométrico.

- Medir la dirección de las diaclasas.

- Recoger muestras litológicas para su posterior determinación. 


\section{BIBLIOGRAFÍA}

\section{General}

CAstany, G., 1971: Tratado práctico de las aguas subterráneas. Ed. Omega, $672 \mathrm{p}$.

GEZE, B., 1968: La espeleología científica. Barcelona, Colección Microcosmo, $192 \mathrm{p}$.

JULIBERT, M., 1957: «Morfologia Kárstica». Rev. Speleon, Barcelona n. ${ }^{\circ}$ 1-2-3-4, tomo VIII.

LLOPIS LLADO, N., 1952: "Sobre algunos principios fundamentales de morfologia e hidrología kárstica». Rev. Speleon, Barcelona n. ${ }^{\circ} 1-2$, tomo III, p. 33-70.

-1970: Fundamentos de hidrogeología kárstica. Madrid, Ed. Blume, 269 p.

Montoriol, J., 1950: «El Campo de dolinas del Pla de Campgrás (macizo de Garraf, Barcelona)". Rev. Speleon, Barcelona, n. ${ }^{\circ}$ 2, p. 95112.

-1956: "Contribución al conocimiento hidrogeológico del borde oriental del Macizo de Garraf». Rev. Speleon, Barcelona, n. ${ }^{\circ}$ 1-2-3-4, p. 336.

NicoD, J., 1972: Pays et paysages du calcaire. París, Ed. Presses Universitaires de France, 239 p.

PezZI, M., 1977: «Morfología kárstica del sector central de la Cordillera Subbética". Cuadernos de Geografía de la Univ. de Granada, n. 2.

Renault, P., 1971: La formación de las cavernas. Barcelona, Ed. OikosTau, n. ${ }^{\circ} 60,122 \mathrm{p}$. 
Trombe, F., 1974: La Espeleología. Barcelona, Ed. Oikos-Tau, n. ${ }^{\circ} 106$, $121 \mathrm{p}$.

-1977: Las aguas subterráneas. Barcelona, Ed. Oikos-Tau, 134 p.

\section{Específica}

Dabrio González, C. J., 1973: “Geología del sector del Alto Segura». Tesis doctorales de la Univ. de Granada, n. ${ }^{\circ} 28$, p. 388.

García Hernández, M. y López Garrido, A. C., 1979: Itinerarios geológicos en las zonas externas de las Cordilleras Béticas. (Itinerarios Sierras de Cazorla y Segura). Univ. de Granada, 77 p.

GEZE, B., 1974: «Relations entre les phénoménes karstiques de surface et de profondeur". Mémoires et Documents, París, CNRS, tomo II, 195-207 p.

Hernanz, A., 1968: “Consideraciones en torno a los poljes kársticos». Cuadernos de Espeleología, Santander, n. 3 , 31-51 p.

LÓPEZ LIMIA, B., 1985: “Geomorfología del karst de Pinar Negro (Sierra de Segura-Jaén)». Tesis de Licenciatura, Univ. de Murcia, inédita.

SwEetiNG, M., 1973: Karst landforms. Columbia University Press New York, $362 p$. 